LA-UR- 08-7992

Approved for public release;

distribution is unlimited.

Title:

(U) Using Ancillary Information to Improve Hypocenter Estimation: Bayesian Single Event Location (BSEL)

Author(s):

Dale N. Anderson, EES-17

Intended for:

PAGEOPH Pure and Applied Geophysics

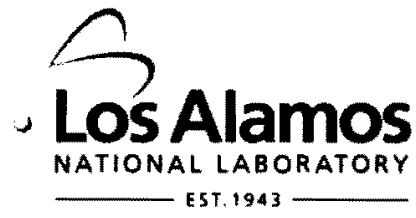

Los Alamos National Laboratory, an affirmative action'equal opportunity employer, is operated by the Los Alamos National Security, LLC for the National Nuclear Security Administration of the U.S. Department of Energy under contract DE-AC52-06NA25396. By acceptance of this article, the publisher recognizes that the U.S. Government retains a nonexclusive, royalty-free license to publish or reproduce the published form of this contribution, or to allow others to do so, for U.S. Government purposes. Los Alamos National Laboratory requests that the publisher identify this article as work performed under the auspices of the U.S. Department of Energy. Los Alamos National Laboratory strongly supports academic freedom and a researcher's right to publish; as an institution, however, the Laboratory does not endorse the viewpoint of a publication or guarantee its technical correctness.

Form $836(7 / 06)$ 


\title{
Using Ancillary Information to Improve Hypocenter Estimation: Bayesian Single Event Location (BSEL) Short title: Bayesian Single Event Location (BSEL)
}

\author{
Deborah K. Fagan, \\ Pacific Northwest National Laboratory, \\ PO Box 999 \\ Richland, WA 99352, \\ dfagan@pnl.gov \\ Fax: 509-375-2522 \\ Steven R. Taylor, \\ Rocky Mountain Geophysics, \\ 167 Piedra Loop \\ Los Alamos, NM 87544 \\ Frederick R. Schult, \\ Air Force Research Laboratory, \\ 29 Randolph Rd \\ Hanscom AFB, MA 01731 \\ Dale N. Anderson, \\ Los Alamos National Laboratory, \\ Earth and Environmental Sciences \\ Los Alamos, NM 87545
}

April 14, 2008 


\begin{abstract}
We have developed and tested an algorithm, Bayesian Single Event Location (BSEL), for estimating the location of a seismic event. The main driver for our research is the inadequate representation of ancillary information in the hypocenter estimation procedure. The added benefit is that we have also addressed instability issues often encountered with historical NLR solvers (e.g., non-convergence or seismically infeasible results). BSEL differs from established nonlinear regression techniques by using a Bayesian prior probability density function (prior PDF) to incorporate ancillary physical basis constraints about event location. $P$-wave arrival times from seismic events are used in the development. Depth, a focus of this paper, may be modeled with a prior PDF (potentially skewed) that captures physical basis bounds from surface wave observations. This PDF is constructed from a Rayleigh wave depth excitation eigenfunction that is based on the observed minimum period from a spectrogram analysis and estimated near-source elastic parameters. For example, if the surface wave is an $R g$ phase, it potentially provides a strong constraint for depth, which has important implications for remote monitoring of nuclear explosions. The proposed Bayesian algorithm is illustrated with events that demonstrate its congruity with established hypocenter estimation methods and its application potential.

The BSEL method is applied to three events: 1) a shallow Mw 4 earthquake that occurred near Bardwell, KY on June 6, 2003, 2) the Mw 5.6 earthquake of July 26, 2005 that occurred near Dillon, MT, and 3) a deep Mw 5.7 earthquake that occurred off the coast of Japan on April 22, 1980. A strong $R g$ was observed from the Bardwell, KY earthquake that places very strong constraints on depth and origin time. No $R g$ was actually observed for the Dillon, MT earthquake, but we used the minimum observed period of a Rayleigh wave ( 7 seconds) to reduce the depth and origin time uncertainty. Because the Japan event was deep, there is no observed surface wave energy. We utilize the prior generated from the Dillon, MT event to show that even in the case when a prior is inappropriately applied, high quality data will overcome its influence and result in a reasonable hypocenter estimate.
\end{abstract}

keywords: seismic event location, Bayesian methods, maximum likelihood

\title{
1 Introduction
}

Most current single event hypocenter algorithms in use follow Geiger (1912), which utilizes nonlinear regression (NLR) theory (Menke, 1989; Seber and Wild, 1989; Casella and Berger, 1990). Given accurate $P$-wave arrival time picks from event waveforms, single event hypocenter accuracy is fundamentally 
dependent on the bias and uncertainty of travel time models and the configuration of stations observing an event (Flinn, 1965). It is well known in seismology that oftentimes depth and origin time cannot be independently resolved with NLR methods even with many realistic configurations of station locations. For example, travel time residuals from earthquakes observed only by distant stations have a nearly perfect trade-off between the origin time and depth, that is, origin time and depth are unidentifiable parameters in the NLR model. Klose and Seeber (2007) recently concluded that approximately $88 \%$ of shallow earthquakes in stable continental regions may be deeper than estimated and suggested that the reason is poor station configuration. Even with good station configuration, NLR inference assumes that the travel time models are of sufficient quality so that hypocenter estimation bias is small. Some solver algorithms for NLR provide depth estimates (and associated ellipsoid confidence regions) that can be inconsistent with the Earth's heterogeneous structure. It is not unusual for current location algorithms to return a hypocenter solution with event depth above the surface of the earth or well below known limits of seismicity. In the former case a geophysicist typically assigns a very shallow depth to the event; in the latter case the solution is statistically valid and the confidence bounds are large enough to encompass more realistic depths, but they are inconsistent with realistic bounds on event depth.

Our proposed unifying hypocenter algorithm, Bayesian Single Event Location (BSEL), simply places physical basis constraints on an event hypocenter with a prior PDF. BSEL incorporates many features that are more general than current methods but that are still familiar to seismologists. The nonlinear behavior of travel time models is reflected in the results because BSEL does not linearize the problem, as NLR does. Other methods which do not rely on linearization of the nonlinear travel time model include genetic algorithms (Sambridge and Gallagher, 1993; Billings et al., 1994), the neighborhood algorithm (Sambridge and Kennett, 2001), simulated annealing (Billings, 1994), and pattern recognition (Nicholson et al., 2002).

BSEL uses the likelihood function to evaluate travel time residuals, therefore any probabilistic model for the residuals can be incorporated and Gaussian residuals are not a requirement. Flexibility in the incorporation of a diversity of ancillary information into the hypocenter calculation is also unique. We illustrate the concept with the detection of short period surface waves but any additional useful information regarding hypocenter parameters may be similarly incorporated. Examples include incorporation of $S$ and $P$ wave arrival times, known locations of faults, historical knowledge of event locations, information from satellite imagery, or, in the case of nuclear explosion monitoring, the location of known test sites. 
The Bayesian approach to hypocenter estimation presented here is relevant for forensic analysis, however we believe that the method could be extended to routine nuclear explosion monitoring as well. In this paper, we illustrate the BSEL method with a physically-based depth prior directly constructed from the minimum observed period of a surface wave. Results from three events are discussed. The first event is a shallow earthquake that occurred near Bardwell, KY with a 1.5-second, high confidence, observed $R g$ phase, a strong indication of shallow depth. The second event is a shallow earthquake near Dillon, MT that does not have an $R g$ phase but has a Rayleigh wave phase with a minimum observed period of 7 seconds. The third event is a deep earthquake off the coast of Japan that has no observed surface wave energy. We use this event to demonstrate that BSEL is congruous with established hypocenter estimation methods.

\section{BSEL Model Formulation and Hypocenter Estimation}

We develop BSEL from $P$-wave arrival times, however the methods can be generalized to other hypocenter-defining waveform measurements. The basic single event arrival time model is

$$
t_{i}=\tau+T\left(\boldsymbol{\theta}_{0}, \boldsymbol{\theta}_{i}\right)+\epsilon_{i}=F\left(\tau, \boldsymbol{\theta}_{0}, \boldsymbol{\theta}_{i},\right)+\epsilon_{i}=F\left(\Theta_{0}, \boldsymbol{\theta}_{i},\right)+\epsilon_{i}, \quad i=1,2, \ldots, n
$$

(Stein and Wysession, 2003, Chap. 7), where $t_{i}$ is the arrival time of the $P$-wave at the $i^{\text {th }}$ station, $\tau$ is the event origin time, $T$ is the travel time from the event located at $\theta_{0}=[x, y, z]^{T}$ to the station located at $\theta_{i}=\left[x_{i}, y_{i}, 0\right]^{T}$ and $\epsilon_{i}$ is a Gaussian random variable with zero mean and variance $\sigma^{2}$, where $\epsilon_{i}$ is independent of $\epsilon_{j}$ for all $i \neq j$. Here $\Theta_{0}$ is the event hypocenter; that is, $\Theta_{0}=[x, y, z, \tau]^{T}$ and $F\left(\Theta_{0}, \theta_{i}\right)$ is nonlinear. Gradient solvers expand Equation (1) using a first-order Taylor series and iteratively solve for $\Theta_{0}$ (Menke, 1989).

NLR is an established approach for hypocenter estimation and there are many optimizing algorithms which will solve for $\Theta_{0}$. However because of the severe tradeoff between depth and origin time, with gradient-based solvers for NLR it is possible to estimate the depth $(z$ ) as a negative number (an airquake), or to get an unreasonable estimate of the depth given known seismicity.

BSEL combines the model in Equation (1) with a probability model of the parameters $\Theta_{0}$ to form the joint probability model but does not linearize with a first-order Taylor series. Implicit in the development is the concept that the parameters $\tau$ and $\theta_{0}$ are random with an a priori defined probability density function (PDF). In this paper, we assume Gaussian residuals, but it is not a requirement. Any 
appropriate probabilistic model for the travel time residuals can be used.

From Equation (1),

$$
t_{i}-\tau-T\left(\theta_{0}, \theta_{i}\right)=\epsilon_{i}, \quad i=1,2, \ldots, n
$$

and if the $\epsilon_{i}$ for an event observed at $n$ stations are modeled as uncorrelated (independent) $N\left(0, \sigma^{2}\right)$ random variables, then theory gives the joint probability model of the residuals $\epsilon_{1}, \epsilon_{2}, \ldots, \epsilon_{n}$ as the product of $N\left(0, \sigma^{2}\right)$ PDFs. The argument $\epsilon_{i}$ in the density functions is then replaced with $t_{i}-\tau-$ $T\left(\boldsymbol{\theta}_{0}, \boldsymbol{\theta}_{i}\right)$, giving the joint probability model of the station arrival times $\boldsymbol{t}$ conditional on $\boldsymbol{\Theta}_{0}$ as

$$
f\left(\boldsymbol{t} \mid \Theta_{0}\right)=\prod_{i=1}^{n} \phi\left(\frac{t_{i}-\tau_{0}-T\left(\theta_{0}, \theta_{i}\right)}{\sigma}\right)
$$

where $\phi(\cdot)$ is the standard normal PDF evaluated at the value inside the parenthetical. Equation (3) is the likelihood of the model parameters $\Theta_{0}$.

BSEL combines Equation (3) with ancillary information regarding the location parameters. As discussed in Section 3, if high quality surface wave energy is detected in one or more waveforms, then the associated event could be shallow. If the observed surface wave energy is an $R g$ phase, then then event has a high probability of being very shallow. A priori PDF for depth with the majority of the mass between, say, 0 and $10 \mathrm{~km}$ and skewed shallow can be combined with the likelihood of $t$ to constrain the estimation of the hypocenter to a shallow depth. The prior PDF for $\Theta_{0}$ is denoted $\pi\left(\Theta_{0}\right)$ and the joint PDF of the data and the hypocenter parameters is

$$
f\left(t, \Theta_{0}\right)=\pi\left(\boldsymbol{\Theta}_{0}\right) f\left(t \mid \Theta_{0}\right)
$$

The BSEL model is simply the posterior PDF of the parameters $\Theta_{0}$ conditional on the data $t$,

$$
f\left(\boldsymbol{\Theta}_{0} \mid \boldsymbol{t}\right)=C(\boldsymbol{t}) \pi\left(\boldsymbol{\Theta}_{0}\right) f\left(\boldsymbol{t} \mid \boldsymbol{\Theta}_{0}\right)
$$

where $C(t)$ is a normalizing constant that ensures the PDF integrates to one. The prior PDF $\pi\left(\Theta_{0}\right)$ is a mathematical representation of physical basis constraints. Equation (5) shows that the posterior is the product of information contained in the prior, $\pi\left(\Theta_{0}\right)$, with information contained in the data, $f\left(t \mid \Theta_{0}\right)$. If the prior contains little information (i.e., is flat or slowly changing) it will have no influence on the resulting posterior distribution. Conversely, if the prior contains a large amount of information (i.e., is 
highly-skewed or quickly changing) it could have a strong influence on the result. The influence of the prior depends on the amount of information in the data and the degree of agreement between the prior and the data.

There are several methods available for solving the BSEL model of Equation (5), including Gibb's Sampling and Monte Carlo integration, to name two. For this demonstration, we simply calculate the numerator of Equation (5) for a 5-dimensional array of pixels $(\Theta)$ and then numerically integrate across the array to estimate $C(t)$.

Section 3 develops examples of $\pi(\cdot)$. From the perspective of Bayesian methods, all information about event hypocenter and origin time $\Theta_{0}$ is contained in the posterior PDF Equation (5). One of two characteristics of the posterior PDF can be used as the hypocenter solution. The first is the average value of $\Theta_{0}$, which provides a hypocenter solution that balances between all possible solutions. This average can be strongly influenced by stations with unusual residuals in the likelihood and yet these stations should not be removed for hypocenter determination. The second characteristic, and the one we use, is the mode of the posterior PDF, which is more robust to the influence of stations with unusual residuals and yet is still sensitive to hypocenter parameter $\pi\left(\Theta_{0}\right)$. This is the value of $\Theta_{0}$ that is most probable given the data $t$. There are theoretical foundations for using the posterior mode for hypocenter and origin time-these are reserved for the Appendix.

\section{Specification of $\pi\left(\theta_{0}\right)$}

We illustrate the concept of incorporating ancillary information into the hypocenter estimation problem by considering the observation of surface wave energy. If observed under the right circumstances, it can be used as a basis for building a prior distribution for depth. The best possible case occurs in the presence of an unambiguous $R g$ detection. Because $R g$ is a fundamental mode Rayleigh wave, it is present only in waveforms for very shallow events, typically with focal depths $<3 \mathrm{~km}$. Mining, earthmoving, and construction explosions, as well as very shallow earthquakes, are examples of events that can generate $R g$ energy. However, $R g$ propagation can be seriously affected by shallow structure and is not typically observed at epicentral distances greater than about $200 \mathrm{~km}$, if at all (e.g., Kafka, 1990). Typically, $R g$ gets scattered by surface heterogeneity and topography over relatively short distances (e.g., 10's to $200 \mathrm{~km}$ ). Fortunately, $R g$ is not solely required to build our prior distribution for depth. As we will show below, observation of slightly longer period surface waves introduces some constraint for shallow depth, particularly for small events having few first arrival time picks. Utilization of the 
minimum observed surface wave period will provide useful bounds on the estimated depth.

Consider the plot shown in Figure 1. It is a two-dimensional plot showing seismic amplitude (or energy) as a function of group velocity versus period and an estimate of the near surface elastic wave velocity. To compute these plots, we use the multiple-filter technique of Landisman et al. (1969). A broadband seismogram is run through a series of narrow-band filters. It is assumed that each band passed trace represents the real part of a complex waveform and that the imaginary part is the Hilbert transform of the filtered signal (e.g., Oppenheim and Shafer, 1975). Depending on the dispersive characteristics of the medium, the group arrival time is approximately equal to the time of maximum amplitude of the envelope of the complex filtered signal. We use a phaseless Gaussian filter that gives the optimum trade off between timed and frequency resolution. An envelope function is computed from the square root of the sum of squares of the real and imaginary traces.

Insert Figure 1 here.

Knowing the distance between the earthquake and the station, we can convert the time axis of the spectrogram to that of group velocity, from which we can visualize the dispersion (group arrival time) and amplitude at each frequency. Figure 2 shows Rayleigh wave depth excitation functions in a Poisson half space for four different periods and compressional velocities (e.g., Lay and Wallace, 1995, Chap. 4.2). The eigenfunction for this simple case can be represented as the sum of two decaying exponential functions. These functions are smooth, skewed, convex functions which can be easily transformed to a probability density function for depth - our needed prior distribution. The largest observed amplitude and group velocity for an event are used to generate a function similar to one shown in Figure 2. The function is standardized so that it integrates to one, and all of the criteria for a proper probability density function are met. Examination of Figure 2 shows that Rayleigh wave eigenfunctions are more peaked at shorter periods and for explosions detonated in low-velocity media. Shorter-period, lower-velocity functions result in higher-skewed prior distributions for $z$, which match physical basis. The definition of minimum observed period is subjective at this point, but could easily be quantified in future work for operations.

\section{Insert Figure 2 here.}

There are several other physical characteristics that could be used to build a prior distribution for origin time and/or depth. In certain situations, sharp S-wave arrival times can be observed at close distances and can help resolve depth and origin time tradeoffs (see Boyd et al., 1984). Accurate $S$ arrival time picks recorded within approximately a distance of 1.4 times the actual focal depth can provide a powerful constraint on depth. Constraints on the origin time (and hence depth) of an event can be 
achieved using $S-P$ arrival times from Wadati diagrams (e.g., Lay and Wallace, 1995) or by inclusion of S-wave arrival times into the likelihood statement. Inclusion of S-waves requires knowledge of Poisson's ratio or accurate shear-velocity models that is often problematic. Picking S-wave arrival times can be difficult and Gomberg et al. (1990) conclude that incorrectly timed S-waves can result in significant depth errors, so careful consideration is important when forming a prior distribution based on S-wave arrivals.

As noted above, a common seismic indicator for the identification of a shallow source is the presence of the short-period surface wave, $R g$. However, $R g$ is often not observed seismically, because it is very sensitive to the details of the structure of the upper crust. In contrast, infrasound signals can be generated by near-source pumping of the atmosphere by $R g$ from shallow sources. Therefore, an seismo-acoustic detection is generally an indicator of a shallow (or surface) source. In other words, an infrasound signal associated with a corresponding seismic signal may act as a surrogate for an $R g$ detection (see Mutschlecner and Whitaker, 2005, for discussion).

A prior can also be specified for the epicenter ( $x$ and $y$ ) of an event using ancillary information and could be skewed, bell-shaped, or uniform. One could envision a prior for $x, y$, and $z$ that is quite complex, including correlation structure based on known geophysical or geographical constraints. For the analysis presented in this paper, we use a very simple prior for hypocenter to demonstrate the concept. Our prior has no dependence structure between $x, y$, and $z$. For the epicenter, we use a rectangular region of a reasonable size to bound $x$ and $y$ with each epicenter point being equally likely. We divide this rectangular region into square pixels of relatively small size and take the center of the pixel as the candidate epicenter.

We model the hypocenter parameters, origin time, and model variance in Equation (1) as independent, so that the prior for all five parameters is specified as the product of individual priors: $\pi(x) \pi(y) \pi(z) \pi\left(\tau_{o}\right) \pi(\sigma)$. The prior for origin time $\tau_{0}$ is uniform centered on the published origin time.

The estimation of $\sigma^{2}$ has posed problems in uncertainty estimation and the calculation of coverage ellipses from the start. Both Flinn (1965) and Evernden (1969) derive parametric equations in conjunction with NLR solvers to calculate coverage ellipses. Each is known to overstate the uncertainty (and, therefore, the size) of a coverage ellipse. Jordan and Sverdrup (1981) advocate using an inverse- $\gamma$ distribution with hyperparameter $K$ for a $\sigma^{2}$ prior as a method to properly reflect individual observational uncertainty. To avoid the need for hyperparameters at this stage, we use the improper prior $1 / \sigma$. This form does not constrain the variance estimate beyond that estimated in the likelihood and ensures that 
the variance estimate is positive. In this way, the variance estimate is entirely based on the data, with no prior influence and no required determination of hyperparameters.

Computationally BSEL is a more general representation of the single event location problem than NLR methods. It can always be formulated to prevent pathological problems with NLR solvers. Damping methods can be applied to minimize the effect of a steep gradient in NLR solvers; however these constraints are inherent in the BSEL formulation. With a no-constraint prior on $\Theta_{0}$, BSEL gives estimates from quadratic loss that are equivalent to the maximum likelihood estimates of NLR. From NLR analysis, hypocenter and origin time are calculated solely from $P$-wave arrivals, while with BSEL these calculations include ancillary information that can greatly improve estimates.

\section{Results}

We chose three events on which to test the BSEL method. The two shallow quakes we selected illustrate the formulation of the depth prior based on observed Rayleigh wave energy and its usefulness in constraining depth. The deep event does not have any observed Rayleigh wave excitation but we chose it as well to provide an example of how the posterior distribution for depth could be affected when an incorrect prior distribution is supplied.

Two different prior distributions for depth were used for each of these events. The first depth prior (uniform) provides maximum likelihood results, which are consistent with NLR estimates. The second depth prior (skewed) is that based on the physics regarding observed surface wave energy discussed in Section 3 .

To compare uncertainty in our hypocenter estimates, we follow Berger (1985, Chap. 4.3) in the construction of an approximate $(1-\alpha) * 100 \%$ uncertainty region, which we call a posterior density region (PDR), or credible set. First, we find the set

$$
L(k)=\{(\hat{z}, \hat{\tau}): f(\hat{z}, \hat{\tau} \mid t) \geq k\}
$$

Then we calculate the probability of $L(k)$ by

$$
P_{f(\hat{z}, \hat{\tau} \mid t)}(L(k))=\int_{L(k)} f(\hat{z}, \hat{\tau} \mid t) d \hat{z} d \hat{\tau}
$$

The maximum $k$ such that $P(L(k)) \leq(1-\alpha)$ marks the boundary contour of our approximate $(1-\alpha) *$ 
$100 \%$ PDR.

There is an important difference between a posterior density region and a confidence interval. A confidence interval is a coverage interval - it represents the probability that the calculated interval "covers" the true hypocenter, which is fixed but unknown. In a Bayesian framework, the hypocenter is not fixed, but has some space over which it can occur with probabilities indicated by the posterior distribution. So, the posterior density region is the region in which the hypocenter can be located with the specified probability. See Carlin and Louis (2000, Chap. 1.4) for discussion of the different interpretations.

\subsection{Event 1 - Bardwell, KY}

An Mw 4 event occurred near Bardwell, Kentucky on June 6, 2003. The main event and aftershocks were studied by Horton et al. (2005), who estimate an event depth of $2 \mathrm{~km}$. As noted in Langston et al. (2005), the event exhibits high amplitude, long duration surface waves up to $136 \mathrm{~km}$ away, which makes this event useful for our analysis. Figure 3 shows the main event location and the observing stations. We use an AK135 upper mantle model (see Kennett et al., 1995) to compute travel times.

Insert Figure 3 here.

Figure 1 shows the spectrogram and group velocity gram at station WVT located $134 \mathrm{~km}$ from the Bardwell earthquake. Strong surface wave energy can be observed between 1 and 4 seconds period. There is a hint of a group velocity minimum at approximately 2.5 seconds. Using a half-space model and calculating the eigenfunction with a velocity of $4.4 \mathrm{~km} / \mathrm{s}$ we get the prior shown in Figure 4 . This prior has a shape similar to the blue curve shown in the top left panel of Figure 2, and has a peak at approximately $1 \mathrm{~km}$.

Insert Figure 4 here.

Eighty-five stations reported observations to the US Geological Survey. Figure 5 shows the posterior when the skewed depth prior corresponding to Figure 4 is used The black line indicates the $95 \%$ posterior density region, which is the region that contains the true depth and origin time with $95 \%$ probability. The posterior probability is zero over most of the range we investigated, with a strong peak near the surface. The trade-off between depth and origin time is evident. The posterior mode (the most likely value) occurs at $0 \mathrm{~km}$ and 1 second before the published origin time.

Insert Figure 5 here.

Table 1 shows the published and BSEL-calculated parameter estimates using independent uniform 
priors as discussed in Section 2, which is equivalent to calculating the maximum likelihood result. Note that the longitude and latitude estimates are identical and that depth and origin time differ slightly. Two reasons for the slight differences between depth and origin time are that we use an AK135 model without any regional corrections to calculate travel times and our pixelation of the depth axis is a single kilometer, limiting the precision of our estimates. Figure 6 shows the depth/origin time posterior when a uniform depth prior is used. The $95 \%$ posterior density region is larger than that shown in Figure 5 , as expected.

Insert Table 1 here.

Insert Figure 6 here.

To analyze the effect of poor station coverage and the interaction between the prior and the likelihood, we randomly selected 350 samples with $n=6$ stations, requiring an azimuthal gap no larger than $180^{\circ}$ and recalculated the location estimate. Under this scheme we expect the posterior mode using the uniform depth prior to have little resolution and the skewed prior to dominate the data heavily, favoring shallow locations. It is evident from Figure 7 that there is little resolution between depth and origin time with only 6 stations and a uniform prior for depth. Depth estimates range between the surface and $81 \mathrm{~km}$ with significant spread over this range. However, as can be seen in Figure 8, when the $R g$-based depth prior is used, the size and extent of the posterior mode is greatly reduced.

Insert Figure 7 here,

Insert Figure 8 here.

\subsection{Event 2 - Dillon, Montana}

The Dillon, MT event on July 26, 2005 was an Mw 5.6 earthquake that does not show evidence of an $R g$ phase but shows that surface wave observations other than $R g$ are useful for constraining an event. The United States Geological Survey (USGS) depth of this event was constrained to be $5 \mathrm{~km}$, and was relocated in Stickney (2005) using a regional velocity model of Zeiler et al. (2005). The relocation and distribution of aftershocks indicate that the earthquake was approximately $10 \mathrm{~km}$ deep. Figure 9 shows the location of the event and the stations used in our analysis. As with the Bardwell event, we explore good and poor station coverage scenarios. Stations within $80^{\circ}$ of the event in Figure 9 were used for the

good coverage results, while only those between $60^{\circ}$ and $80^{\circ}$ (the pink ones) were used to investigate poor coverage results. We used the regional velocity model of Zeiler et al. (2005) over an AK135 upper mantle model (see Kennett et al., 1995) to compute travel times. 
Insert Figure 9 here.

The surface wave energy plot for station WDC located $973 \mathrm{~km}$ west of the event is illustrated in Figure 10, where the red colors show the largest amplitudes. From this plot it can be seen that the largest amplitudes arrive at approximately 8 seconds with a group velocity of approximately $2.8 \mathrm{~km} / \mathrm{s}$. Note that very little energy arrives at periods of less than approximately 7 seconds. Using a half-space model and calculating the eigenfunction with a velocity of $6.5 \mathrm{~km} / \mathrm{s}$, we get the prior shown in Figure 11. Its shape is similar to the curves shown in the lower left panel of Figure 2. Note that this prior is one of the least skewed possible using surface wave energy as a basis and shows its peak at approximately 2 $\mathrm{km}$.

Insert Figure 10 here.

Insert Figure 11 here.

When the location is estimated using a uniform prior with 62 stations within $80^{\circ}$ of the event the BSEL depth estimate is $1 \mathrm{~km}$ with an origin time of $\tau_{0}-1 \mathrm{~s}$, where $\tau_{0}$ is the published origin time see Figure 12). Recall from the introduction to this section that BSEL results when using a uniform prior are equivalent to the maximum likelihood result. As with Bardwell, the trade-off between depth and origin time is evident. A slight curvature is also visible.

Insert Figure 12 here.

The posterior for depth versus origin time using the skewed prior from Figure 11 is shown in Figure 13. Using the skewed prior for depth results in the same depth-origin time estimate as with the uniform depth prior; the prior does not noticeably influence the solution because there are many stations observing the event and the prior is not very peaked. The slight peakedness of the skewed prior for depth brings the tail of the $95 \%$ posterior density region up slightly from the uniform one. The published location is 5 $\mathrm{km}$, but as noted in the previous section, the relocated depth is approximately $10 \mathrm{~km}$, which is well within the posterior density region for both priors.

Insert Figure 13 here.

To research how BSEL performs with poor station coverage, we omitted stations within $60^{\circ}$ observing the event and recalculated the posterior using each depth prior. Although this represents a somewhat unrealistic scenario for this event, it illustrates the power of the BSEL method for an ill-posed problem. Travel times from twenty-one stations remained in the dataset once the "close" ones were removed. Results using the uniform prior are shown in Figure 14 and for the skewed prior in Figure 15. As expected, the influence of the skewed prior is more evident here than in the good coverage case. The 
mode of the posterior (most likely value) under the uniform prior is $0 \mathrm{~km}$ and two seconds before the published origin time. Under the skewed prior, the mode is approximately $z=2 \mathrm{~km}$ and 1.2 seconds before the published origin time. The trade off between origin time and depth again is evident, as well as the decrease in precision. The $95 \%$ posterior density region for the uniform prior extends beyond 40 $\mathrm{km}$ and $\tau_{0}+4 \mathrm{~s}$, whereas the posterior density region for the skewed prior extends to approximately 30 $\mathrm{km}$. The increased size of the posterior density regions when fewer stations in a poor configuration are used reflects the greater uncertainty and large trade off associated with poor station coverage. Another interesting feature is the curvature evident in the uniform posterior density region, which reflects the nonlinear behavior of the travel time models (Kennett et al., 1995). NLR typically results in symmetric, elliptical confidence regions, which do not reflect the true relationship between depth and origin time.

Insert Figure 14 here.

Insert Figure 15 here.

\subsection{Event 3 - Sea of Japan}

We briefly studied a magnitude 5.7 event that occurred off the coast of Japan on April 22, 1980 at a reported depth of $401.6 \mathrm{~km}$ by the International Seismic Centre (ISC). We use this event as a validation of the BSEL technique and to show that when a minimal amount of high-quality data with good coverage are present the specification of an incorrect prior distribution for depth can still result in a reasonable result. We randomly chose $n=7$ stations within $80^{\circ}$ of the event and used BSEL to estimate the location using both uniform and skewed priors for depth. Because no surface energy was observed for this event, we used the skewed prior from the Dillon, MT event described in Section 4.2 as the skewed prior for depth. Table 2 shows that even when an incorrect prior for depth is used, the BSEL result is consistent with published and non-informative prior results. From Figure 11 we see that when surface wave energy is observed at shorter periods in low velocity media that the probability of an event occurring at a depth of $30 \mathrm{~km}$ is essentially zero. This implies that the data must contain a large amount of information about depth in order to overcome the incorrect prior distribution.

Insert Table 2 here. 


\section{SUMMARY AND FUTURE RESEARCH}

The main driver for our research is the inadequate representation of ancillary information in the hypocenter estimation procedure. The added benefit is that we have also addressed instability issues often encountered with historical NLR solvers (e.g., non-convergence or seismically infeasible results). We have shown that by formulating the problem in a Bayesian framework that these difficulties may be overcome. The addition of a constraining prior distribution for the depth parameter, for instance, avoids numerical instabilities sometimes encountered using historical approaches and has the added benefit of providing a posterior density of hypocenter solutions consistent with geophysical theory.

The BSEL method balances the amount of available data with the information supplied in the prior distributions. When low-information priors (flat or slowly changing) are used, the solutions do not differ significantly from nonlinear regression methods. When informative priors and the data are consistent with one another, the result is a smaller uncertainty region. Barring the pathological case of point-mass priors, when the data and priors are inconsistent a larger uncertainty region will result. The size of the region is dependent on the amount of information in the data (e.g., azimuthal coverage, number of stations, magnitude of travel time residuals) and the degree to which the data and priors contain conflicting information.

Often the configuration of seismic stations which observe an event makes estimation of event origin time and depth difficult. Seismic theory states that if short period surface wave energy is observed by any of these stations, then the event must be shallow, thereby constraining the depth (and, hence, origin time). Most relevant is that the strength of the observed energy has a direct relationship with event depth - the eigenfunction indexed by energy strength - and that the eigenfunction can be used to construct a prior for depth. While this method could be useful for any number of applications, we expect it to be particularly useful in nuclear explosion monitoring. Unfortunately, because of the remoteness of nuclear explosions for which we have digital recordings, there is little or no definitive $R g$ recordings on which to test the BSEL methodology.

There are other sources of information which may be used to construct prior distributions for the other hypocenter parameters. For instance, the incorporation of $S$ and $P$ wave arrival times might further constrain depth, as well as origin time. We will research the construction of more sophisticated prior distributions for hypocenter parameters than the one illustrated here - ones which incorporate ancillary information regarding hypocenter parameters in addition to depth. 
Another important contributor to unrealistic estimates of hypocenter is undetected bias in the travel time model. We intend to explore the robustness of the BSEL technique by varying the strength of potential bias errors in comparison with the variation in posterior density shape.

\section{Acknowledgments}

The authors acknowledge the support of the Department of Energy's Office of Nuclear Nonproliferation Research and Development. This work was completed under the auspices of the U.S. Department of Energy by Pacific Northwest National Laboratory under contract DE-AC06-76RLO 1830. Additionally, discussions with Howard Patton are appreciated. We also acknowledge the in-depth comments of four anonymous reviewers and editor Dr. Arthur Snoke who's comments on the original manuscript and rereviewed comments greatly improved the content of this paper. Their hard work is extremely appreciated. 


\section{References}

Berger, J. O. (1985), Statistical Decision Theory and Bayesian Analysis, New York: Chapman \& Hall, 2nd ed.

Bernardo, J. and Smith, A. (2000), Bayesian Theory, England: John Wiley \& Sons, Inc.

Billings, S. D. (1994), "Simulated annealing for earthquake location," Geophysical Journal International, $118,693-706$.

Billings, S. D., Kennett, B. L. N., and Sambridge, M. S. (1994), "Hypocentre location: genetic algorithms incorporating problem-specific information," Geophysical Journal International, 118, 693-706.

Boyd, T. M., Snoke, J. A., Sacks, I. S., and Rodriguez, A. (1984), "High-resolution determination of the benioff zone geometry beneath southern peru," Bulletin of the Seismological Society of America, 74, $559-568$.

Carlin, B. P. and Louis, T. A. (2000), Bayes and Empirical Bayes Methods for Data Analysis, Boca Raton: Chapman and Hall.

Casella, G. and Berger, R. L. (1990), Statistical Inference, Pacific Grove, California: Wadsworth \& Brooks.

Evernden, J. F. (1969), "Precision of epicenters obtained by small numbers of world-wide stations," Bulletin of the Seismological Society of America, 59, 1365-1398.

Flinn, E. A. (1965), "Confidence regions and error determinations for seismic event location," Reviews in Geophysics, 3, 157-185.

Geiger, L. (1912), "Probability method for determination of earthquake epicentres from arrival time only," Bull. St. Louis Univ., 8, 60-71.

Gomberg, J. S., Shedlock, K. M., and Roecker, S. W. (1990), "The effect of $S$-wave arrival times on the accuracy of hypocenter estimation," Bulletin of the Seismological Society of America, 80, 1605-1628.

Horton, S. P., Kim, W., and Withers, M. (2005), "The 6 June 2003 Bardwell, Kentucky earthquake sequence: evidence for a locally perturbed stress field in the Mississippi embayment," Bulletin of the Seismological Society of America, 95, 431-455.

Jordan, T. H. and Sverdrup, K. A. (1981), "Teleseismic location techniques and their application to earthquake clusters in the South-Central Pacific," Bulletin of the Seismological Society of America, $71,1105-1130$.

Kafka, A. (1990), "As a Depth Discriminant for Earthquakes and Explosions: A Case Study in New England," Bulletin of the Seismological Society of America, 80, 373-394.

Kennett, B. L. N., Engdahl, E. R., and Buland, R. (1995), "Constraints on seismic velocities in the Earth from travel times," Geophysics Joumal International, 122, 108-124.

Klose, C. D. and Seeber, L. (2007), "Shallow Seismicity in Stable Continental Regions," Seismological Research Letters, 78, 554-562.

Landisman, M., Dziewonski, A., and Sato, Y. (1969), "Recent improvements in the analysis of surface wave observations," Geophys. J. R. astr. Soc., 17, 369-403.

Langston, C. P., Bodin, P., Powell, C., Withers, M., Horton, S., and Mooney, W. (2005), "Bulk sediment $Q_{p}$ and $Q_{s}$ in the Mississippi embayment, central United States," Bulletin of the Seismological Society of America, 95, $2162-2179$. 
Lay, T. and Wallace, T. (1995), Modern Global Seismology, San Diego: Academic Press.

Menke, W. (1989), Geophysical Data Analysis: Discrete Inverse Theory, New York: Academic Press, revised ed.

Mutschlecner, J. P. and Whitaker, R. W. (2005), "Infrasound from earthquakes," Journal of Geophysical Research, 110, doi:10.1029/2004JD005067.

Nicholson, T., Sambridge, M., and Gudmundsson, O. (2002), "Hypocenter location by pattern recognition," Journal of Geophysical Research - Solid Earth, 107.

O'Hagan, A. (1994), Kendall's Advanced Theory of Statistics: Volume 2B, Bayesian Inference, Arnold Publishers, London, 6th ed.

Oppenheim, A. V. and Shafer, R. W. (1975), Digital Signal Processing, New Jersey: Prentice-Hall.

Sambridge, M. and Gallagher, K. (1993), "Earthquake hypocenter location using genetic algorithms," Bulletin of the Seismological Society of America, 83, 1467-1491.

Sambridge, M. S. and Kennett, B. L. N. (2001), "Seismic event location: nonlinear inversion using a neighbourhood algorithm," Pure and Applied Geophysics, 158, 241-257.

Seber, G. and Wild, C. (1989), Nonlinear Regression, New York: John Wiley \& Sons, Inc.

Stein, S. and Wysession, M. (2003), An Introduction to Seismology, Earthquakes, and Earth Structure, United Kingdom: Blackwell Publishing.

Stickney, M. (2005), "The 26 July, $2005 \mathrm{Mw} 5.6$ Dillon, Montana earthquake," http://serc.carleton.edu/files/earthscoperockies/stickneyposter_1129844125.pdf.

Zeiler, C. P., Stickney, M. C., and Speece, M. A. (2005), "Revised Velocity Structure of Western Montana," Bulletin of the Seismological Society of America, 95, 759-762. 


\section{Appendix}

BSEL chooses an estimate that minimizes the risk of calculating a poor hypocenter and origin time $\Theta_{0}$. Risk is the expected value of a loss function calculated with the posterior PDF (see Berger, 1985; Bernardo and Smith, 2000), where loss mathematically defines a penalty for poor hypocenter and origin time estimates. Therefore, a loss function needs to be specified with BSEL.

We are free to define loss exclusively in terms of the hypocenter, and in this case loss is a function of the estimated hypocenter $(\hat{\boldsymbol{\theta}})$ and the true hypocenter $\left(\boldsymbol{\theta}_{0}\right)$, and is denoted $l\left(\hat{\boldsymbol{\theta}}, \boldsymbol{\theta}_{0}\right)$. Here, no loss is specified for origin time. The hypocenter estimate is the value of $\hat{\theta}$ that minimizes the expected loss (risk), defined as

$$
\text { risk }=\int l(\hat{\Theta}, u) f(\boldsymbol{u} \mid \boldsymbol{t}) d \boldsymbol{u} .
$$

With loss defined only by hypocenter, origin time simply integrates out of Equation (6). One may specify the posterior hypocenter loss function, $l\left(\hat{\theta}, \theta_{0}\right)$, with some flexibility but some common ones are quadratic loss and zero-one loss, which are discussed here in the context of hypocenter estimation. Both of these loss functions lead to appealing mathematical hypocenter calculations with good physical interpretations. Specifying the posterior loss function in turn specifies the estimate which minimizes Equation (6).

Posterior quadratic loss takes the form

$$
l\left(\hat{\boldsymbol{\theta}}, \boldsymbol{\theta}_{0}\right)=\left(\hat{\boldsymbol{\theta}}-\boldsymbol{\theta}_{0}\right)^{\prime} \boldsymbol{H}\left(\hat{\boldsymbol{\theta}}-\boldsymbol{\theta}_{0}\right),
$$

where $\boldsymbol{H}$ is positive definite. Equation (6) is minimized with loss function Equation (7) when $\hat{\theta}$ is the mean vector of the posterior PDF regardless of the numerical form of $\boldsymbol{H}$. This is easily proved by differentiating Equation (6) with respect to $\hat{\theta}$ and equating to zero. The Bayes estimate under quadratic loss is the mean vector of the posterior PDF because this loss function heavily weights the tails of the posterior in determining $\hat{\boldsymbol{\theta}}$. A hypocenter posterior with significant probability in the tail of the PDF can be caused by outlier influence from a subset of seismic stations used to estimate hypocenter. Quadratic loss would be used when the influence of these stations is deemed important regardless of their apparent outlier behavior, that is, high confidence association and arrival time picks regardless of large residuals. In application, the matrix $\boldsymbol{H}$ could be the identity matrix or one of weights as described in Menke (1989), resulting in weighted NLR.

Zero-one loss is typically thought of in terms of a hypothesis test; the loss is zero if the correct hypocenter is calculated and one otherwise. For BSEL, one can consider the candidate hypocenter $\hat{\boldsymbol{\theta}}$ as a rich global grid with each grid point an individual hypothesized location. Suppose the true hypocenter $\boldsymbol{\theta}_{0}$ lies inside a sphere centered at a grid point $\hat{\boldsymbol{\theta}}$. The zero-one loss function is

$$
l\left(\hat{\boldsymbol{\theta}}, \boldsymbol{\theta}_{0}\right)=1-I_{B_{\epsilon}(\hat{\theta})}\left(\theta_{0}\right)
$$

where $B_{\epsilon}(\hat{\boldsymbol{\theta}})$ is a sphere of radius $\epsilon$ centered at a grid point $\hat{\boldsymbol{\theta}}$. If the sphere encloses the hypocenter $\theta_{0}$, the indicator function $I_{B_{e}(\hat{\theta})}\left(\theta_{0}\right)=1$ and the loss is zero; otherwise $I_{B_{\varepsilon}(\hat{\theta})}\left(\theta_{0}\right)=0$. The risk [Equation (6)] is minimized when

$$
\int I_{B_{\varepsilon}(\hat{\boldsymbol{\theta}})}(\boldsymbol{u}) \hat{f}(\boldsymbol{u} \mid \boldsymbol{t}) d \boldsymbol{u}
$$

is maximized. Substituting a grid point $\hat{\theta}$ into Equation (9) reduces the limits of the integral to the sphere around that grid point. For choices of $\hat{\theta}$ in the tail of the posterior PDF [Equation (5)], the integral evaluates to a small value, and thus high risk. For choices of $\hat{\boldsymbol{\theta}}$ near the mode of posterior PDF, the integral evaluates to a relatively large value, which results in relatively low risk. As $\epsilon \rightarrow 0$, the choice for $\hat{\theta}$ giving the lowest risk is the mode of the posterior PDF (see O'Hagan, 1994; Bernardo and Smith, 2000). 
In our development we use zero-one loss and select the posterior mode as an event hypocenter estimate. This has intuitive appeal because the mode is the most probable hypocenter given the station arrival times. When the prior for depth results in a Gaussian posterior, the choice between quadratic and zero-one loss does not impact the hypocenter estimate. However, with a non-symmetric prior for depth, the choice of loss function is important. If $R g$ is present with high confidence, we use a highly shallow-skewed prior for $z$, which, in the absence of a large amount of data, will result in a skewed posterior for depth. In this case, the mode is smaller than the median, which results in a more shallow - and often more realistic - depth estimate. 


\section{Tables}

Table 1: Published and uniform prior BSEL location estimates for the Bardwell event using 85 stations.

\begin{tabular}{|c|c|c|c|c|}
\hline & Longitude & Latitude & Depth $(\mathrm{km})$ & Origin Time \\
\hline Published & -88.97 & 36.87 & 2.5 & $12: 29: 34.00$ \\
\hline BSEL & -88.97 & 36.87 & 2.0 & $12: 29: 32.75$ \\
\hline
\end{tabular}


Table 2: Published and BSEL-estimated values for depth and origin time for the deep event using seven randomly selected stations within $80^{\circ}$ of the published location.

\begin{tabular}{|c|c|c|}
\hline & Depth $(\mathrm{km})$ & Origin Time \\
\hline Published & 401.6 & $5: 34: 14.5$ \\
\hline BSEL - uniform & 410 & $5: 34: 14.0$ \\
\hline BSEL - skewed & 407 & $5: 34: 14.0$ \\
\hline
\end{tabular}




\section{Figure Captions}

Figure 1. The right panel is a group velocity gram showing the seismogram used to compute the spectrogram on the right. The time axis has been altered to group velocity using event and station separation. The left panel is the spectrogram, which plots energy as a function of group velocity and period. Blue colors in the left panel denote small amplitudes; red colors, large amplitudes. This figure originates from station WVT located $134 \mathrm{~km}$ southeast of the Bardwell, KY event discussed in Section 4.1 .

Figure 2. Rayleigh depth excitation functions in a Poisson half space for four different periods and compressional velocities. From the figure, we see that observations of short period Rayleigh waves can place a strong constraint on depth, particularly at shorter periods and in low velocity media.

Figure 3. Station locations of stations having $P$ arrival times (green triangles) for the Bardwell event (marked by a red star). The solid circles mark $20^{\circ}$ increments in epicentral angle.

Figure 4. Prior distribution for depth for the Bardwell event, based on the surface wave characteristics seen in Figure 1. Based on Figure 1, we use a group velocity of $4.4 \mathrm{~km} / \mathrm{s}$ to generate this prior.

Figure 5. Posterior distribution for depth versus origin time for the Bardwell event calculated with $n=85$ stations and the skewed prior for depth based on surface wave observations. Origin times are given relative to the published origin time - zero is the published origin time. The black dot marks the estimated hypocenter.

Figure 6. Posterior distribution for depth versus origin time for the Bardwell event using $n=85$ stations and a uniform prior distribution for depth. Origin times are given relative to the published origin time - zero is the published origin time. The black dot marks the estimated hypocenter.

Figure 7. Distribution of the posterior mode of depth and origin time for the Bardwell event using uniform prior distributions and a sample size of $n=6$ stations. Red shades represent higher probabilities; blue shades represent lower probabilities. Origin times are given relative to the published origin time - zero is the published origin time.

Figure 8. Distribution of the posterior mode of depth and origin time for the Bardwell event using an $R g$-based prior distribution for depth, a uniform prior for origin time, and a sample size of $n=6$ stations. Red shades represent higher probabilities; blue shades represent lower probabilities. Origin times are given relative to the published origin time $(\tau)$. The depth scale of Figure 7 is used to facilitate comparison.

Figure 9. Station locations of stations reporting $P$ arrival times (triangles) for the Dillon 2006 event, marked by a red star. The solid circles mark $20^{\circ}$ degree increments in epicentral angle. Stations within $80^{\circ}$ are used to estimate hypocenter under good station coverage. Magenta stations are those between $60^{\circ}$ and $80^{\circ}$ of the event and are used to estimate hypocenter under the scenario of poor coverage.

Figure 10. Multiple filter analysis for Rayleigh waves recorded at station WDC located $973 \mathrm{~km}$ west of the Dillon, MT earthquake. The right panel is a group velocity gram showing the seismogram at station WDC, where the time axis has been altered to group velocity using event and station separation. The left panel is the spectrogram, which plots energy as a function of group velocity and period. Blue colors in the left panel denote small amplitudes; red colors, large amplitudes. Most of the energy arrives at 
periods between about 7 and 10 seconds and some dispersion is evident.

Figure 11. Prior distribution for depth for the Dillon event, based on the surface wave characteristics seen in Figure 10. Based on Figure 10, we use a group velocity of $6.5 \mathrm{~km} / \mathrm{s}$ to generate this prior.

Figure 12. Posterior distribution for depth versus origin time for the Dillon event using $n=62$ stations and a uniform prior for depth. The dark line is the $95 \%$ posterior density region. Origin times are given relative to the published origin time - zero is the published origin time. The black dot marks the estimated hypocenter.

Figure 13. Posterior distribution for depth versus origin time for the Dillon event using $n=62$ stations and a skewed prior for depth based on Figure 11. The dark line is the 95\% posterior density region. Origin times are given relative to the published origin time - zero is the published origin time. The black dot marks the estimated hypocenter.

Figure 14. Posterior distribution for depth versus origin time for the Dillon event using $n=21$ stations between $60^{\circ}$ and $80^{\circ}$ and a uniform prior for depth. The dark line is the $95 \%$ posterior density region. Origin times are given relative to the published origin time - zero is the published origin time. The black dot marks the estimated hypocenter.

Figure 15. Posterior distribution for depth versus origin time for the Dillon event using $n=21$ stations between $60^{\circ}$ and $80^{\circ}$ and a uniform prior for depth. The dark line is the $95 \%$ posterior density region. Origin times are given relative to the published origin time - zero is the published origin time. The black dot marks the estimated hypocenter. 
Figures 

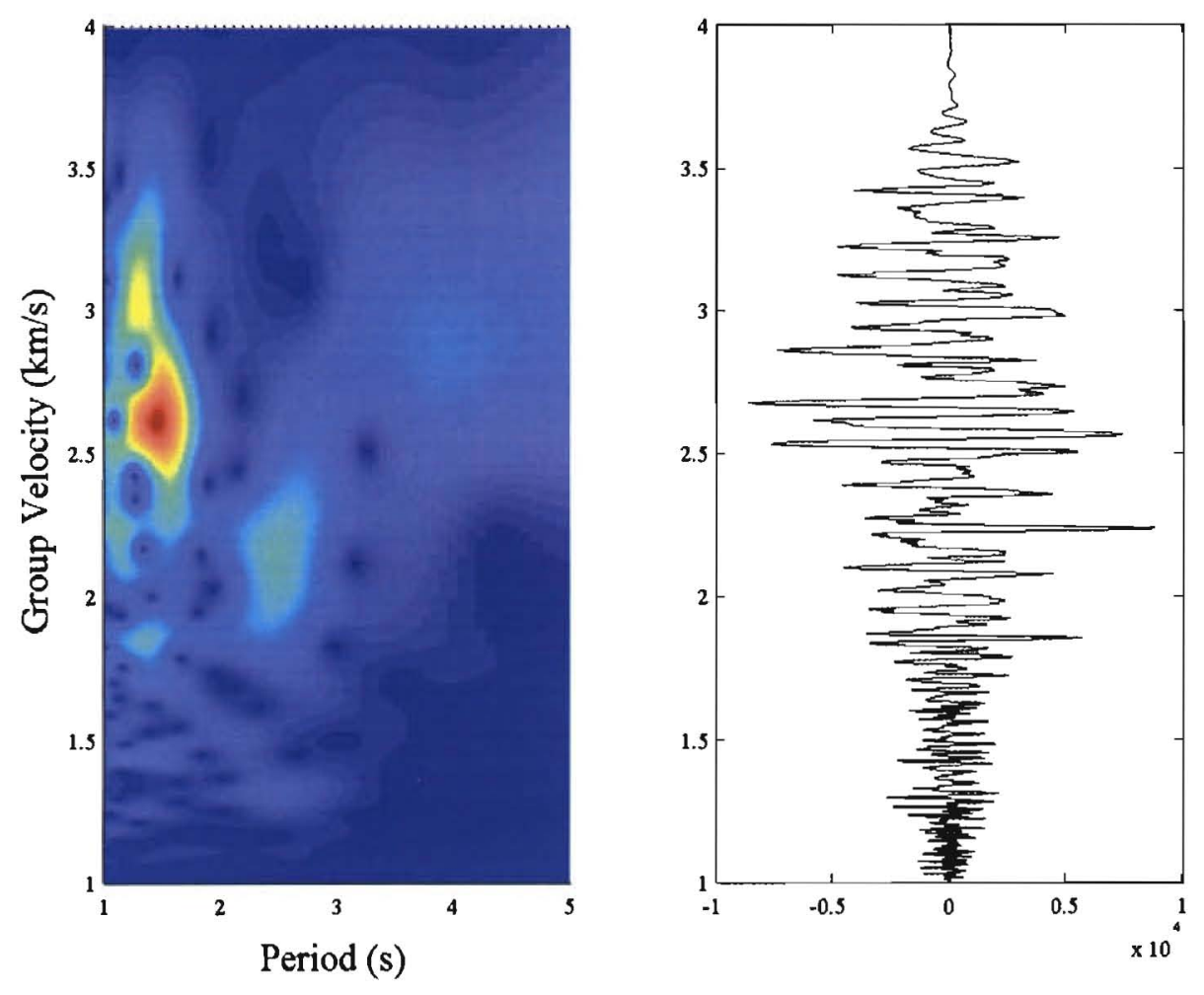

Figure 1: 

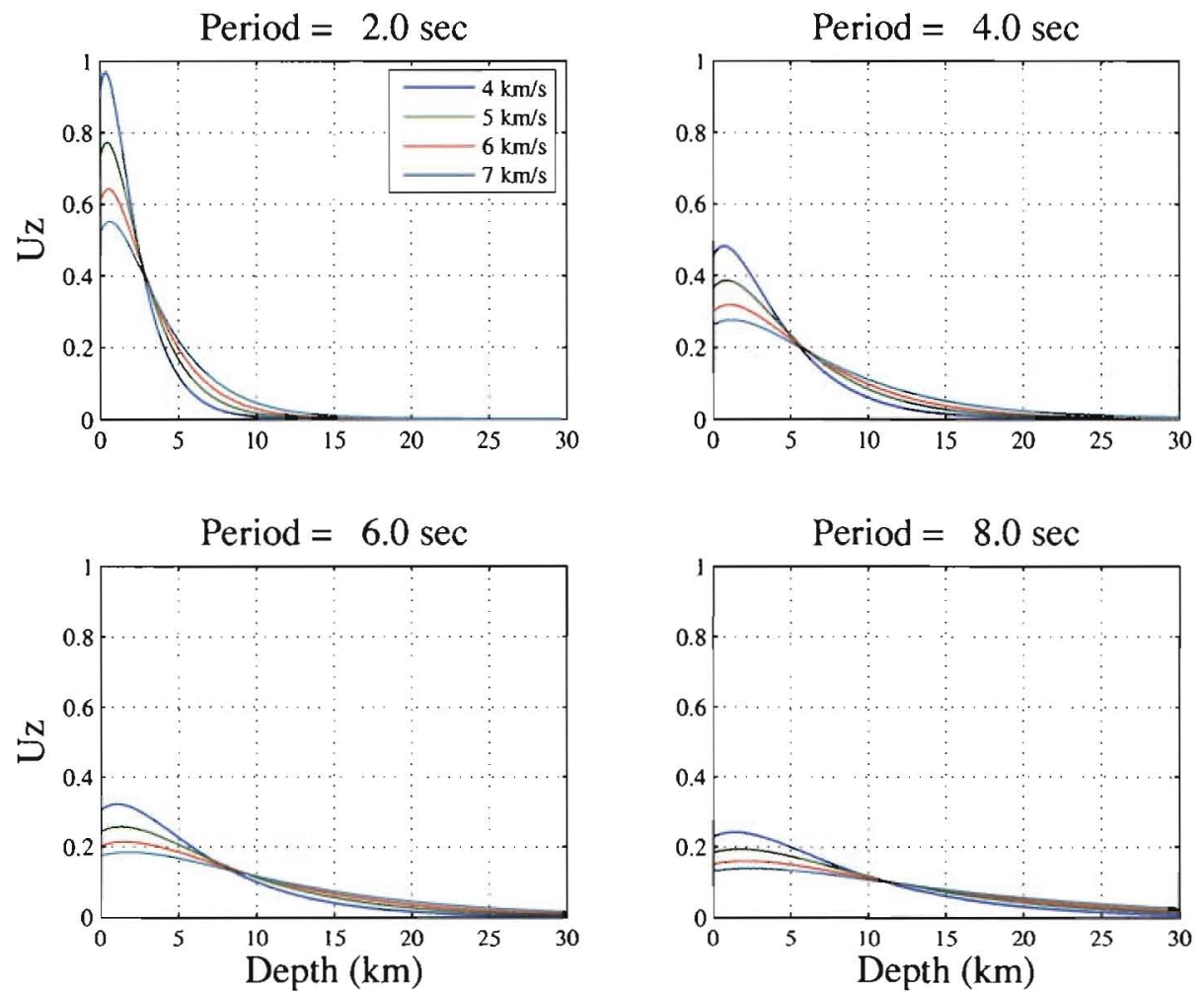

Figure 2: 


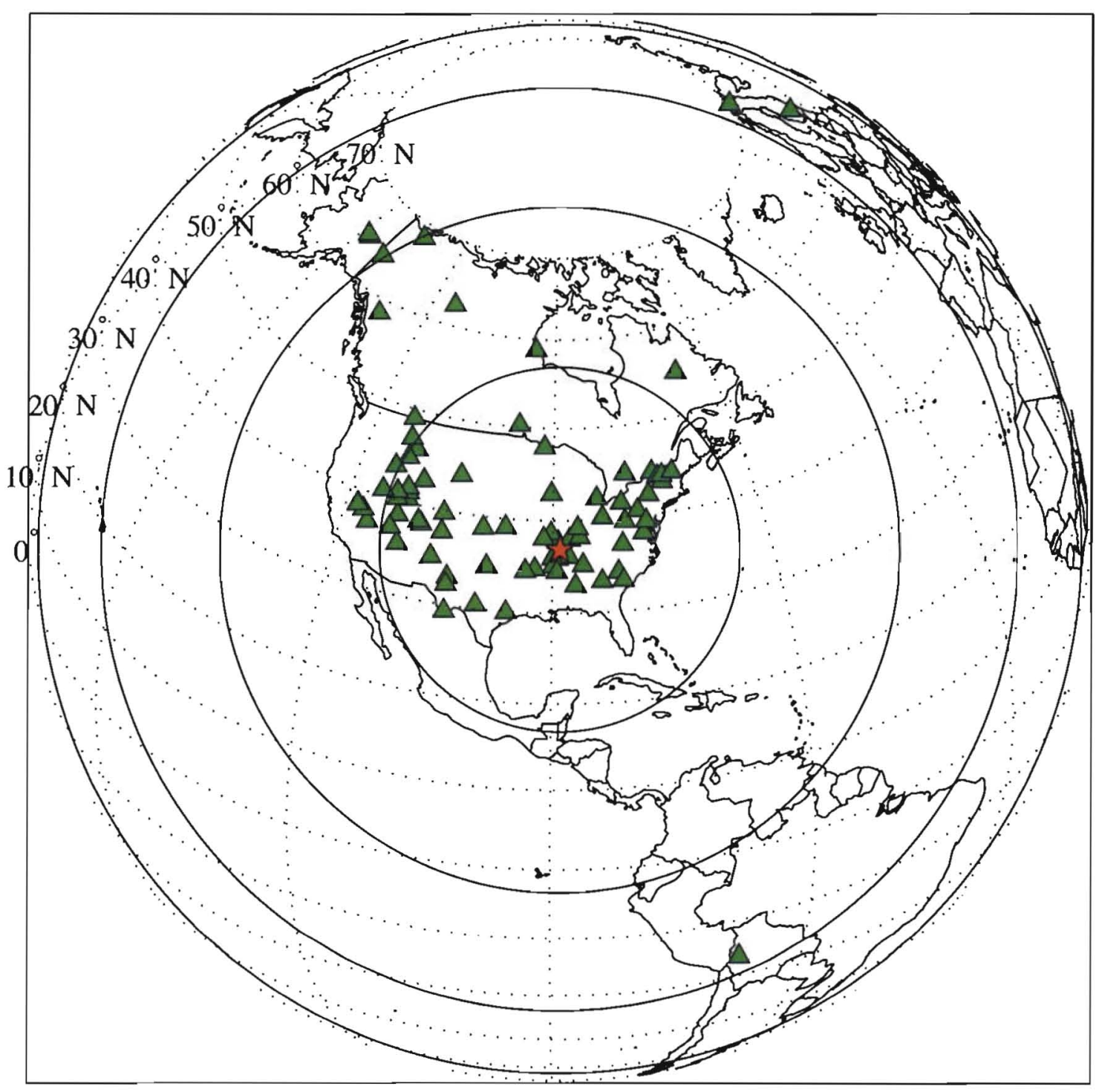

Figure 3: 


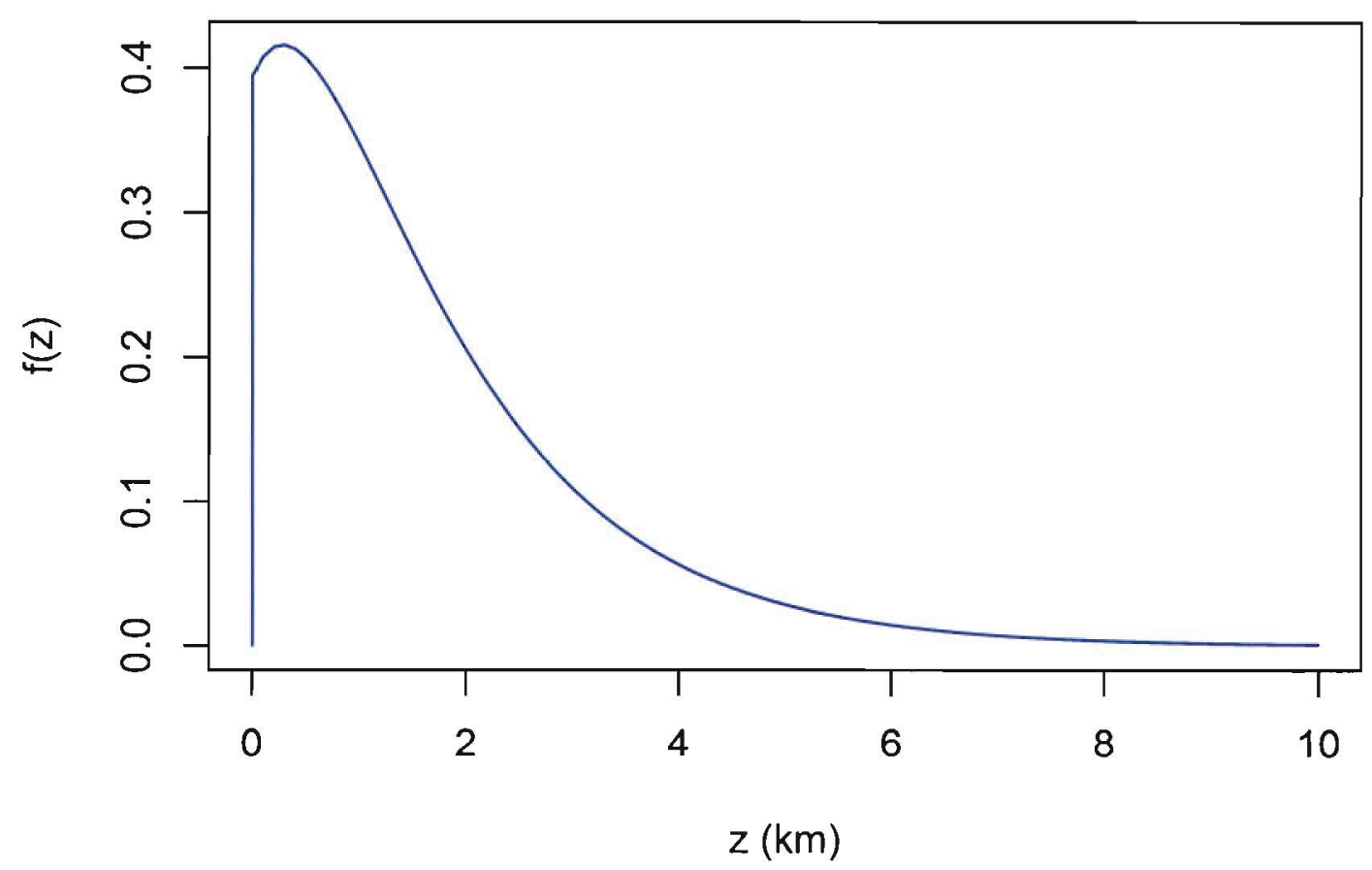

Figure 4: 


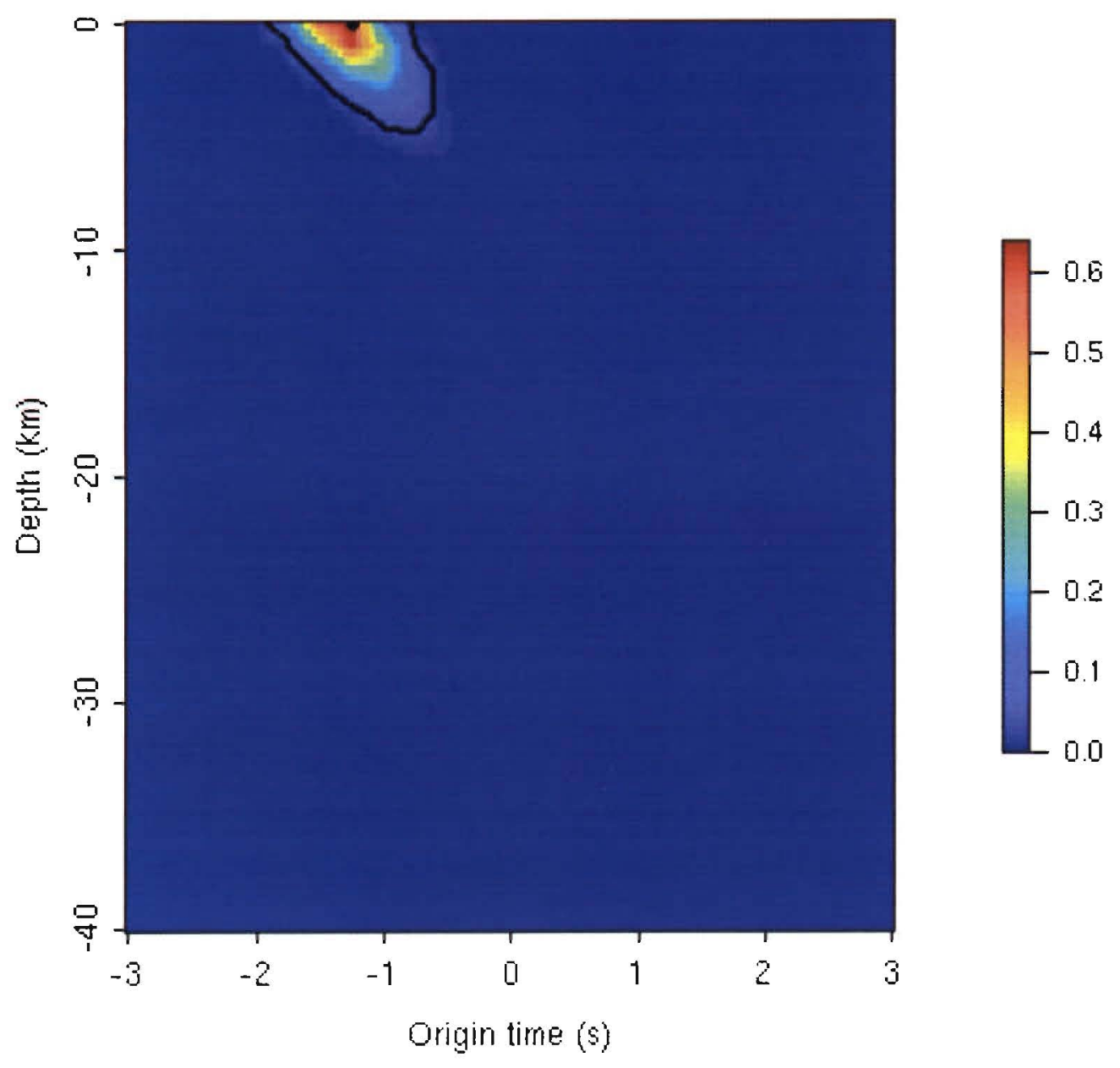

Figure 5: 


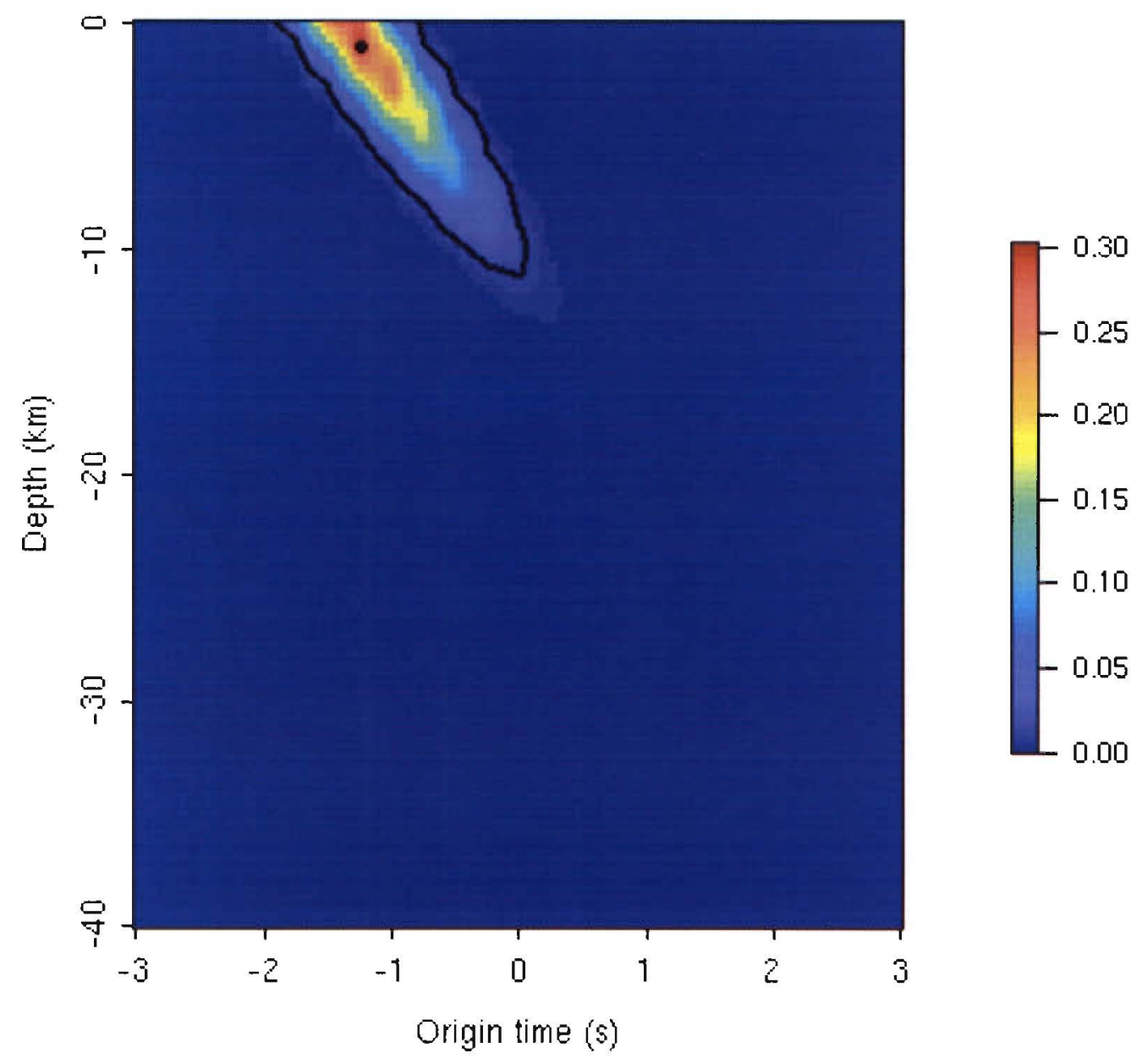

Figure 6: 


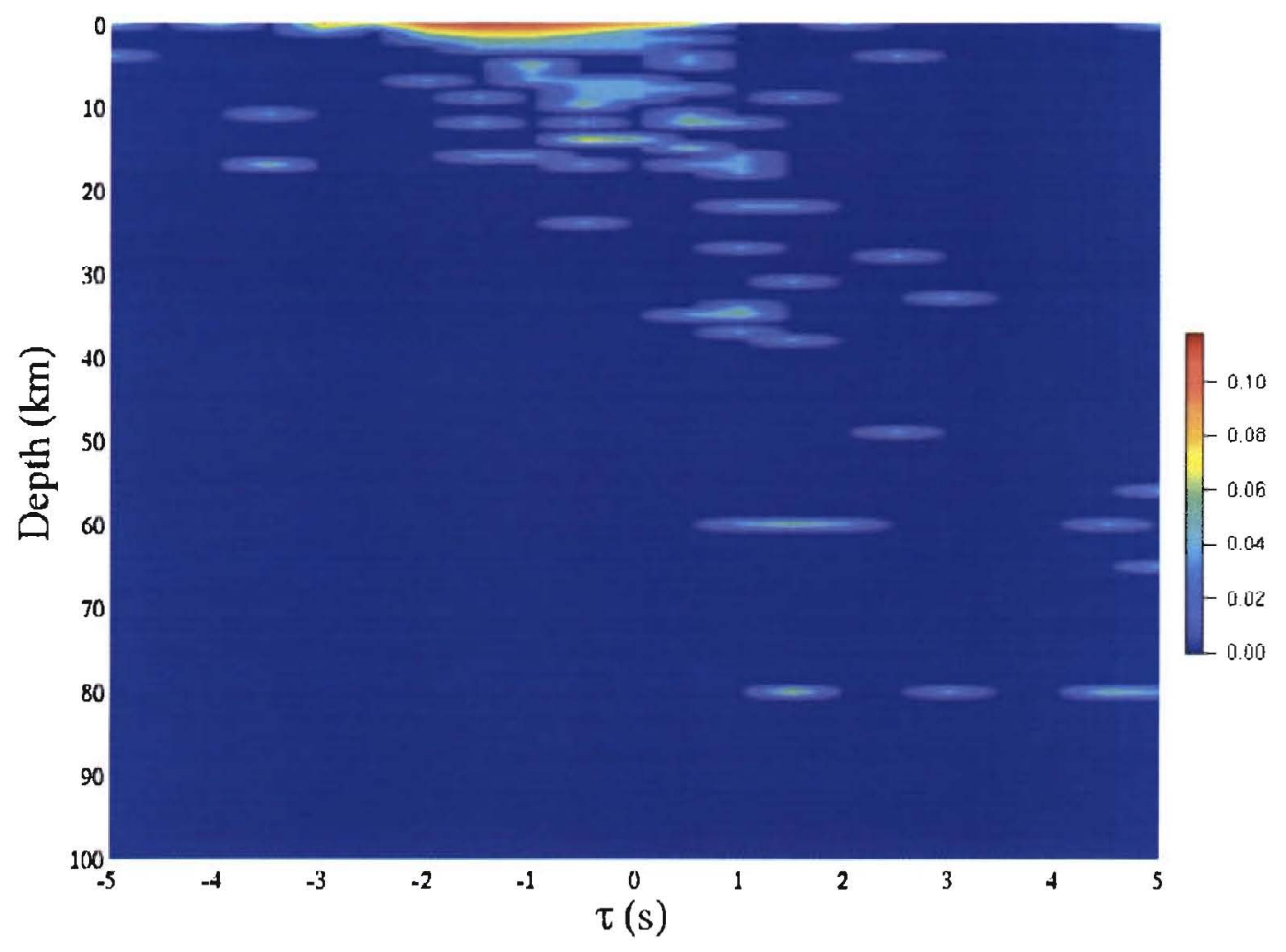

Figure 7: 


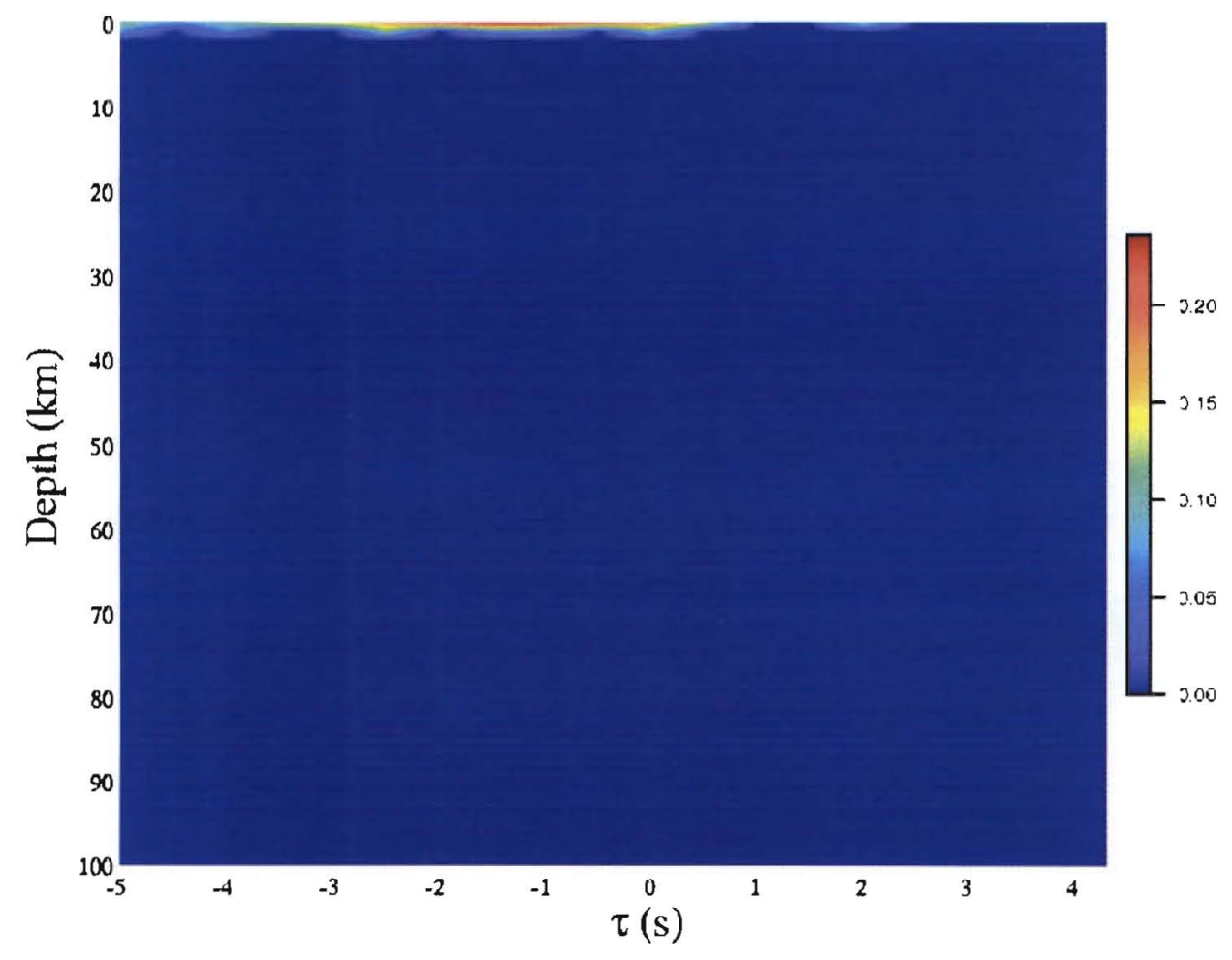

Figure 8: 


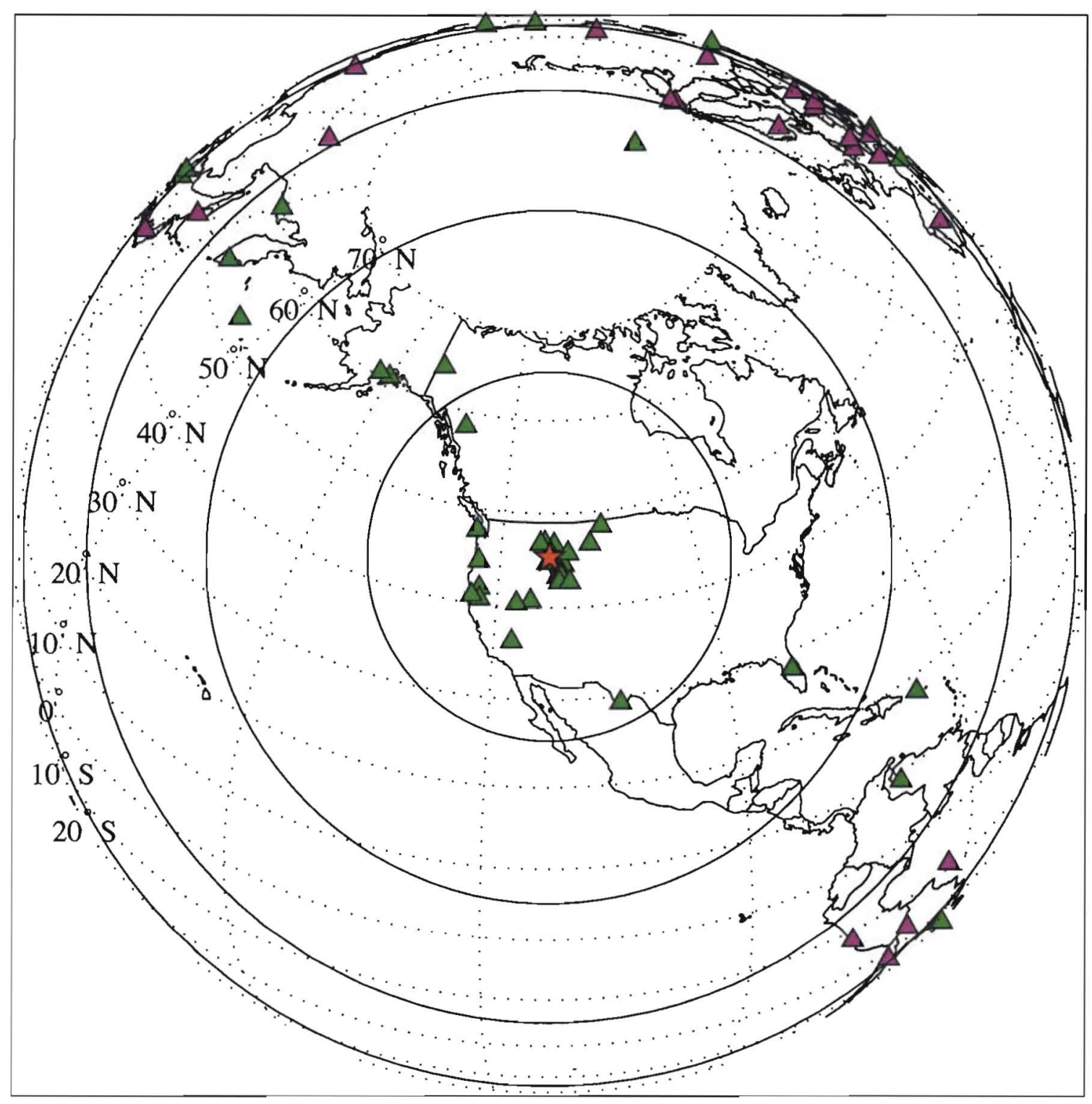

Figure 9: 

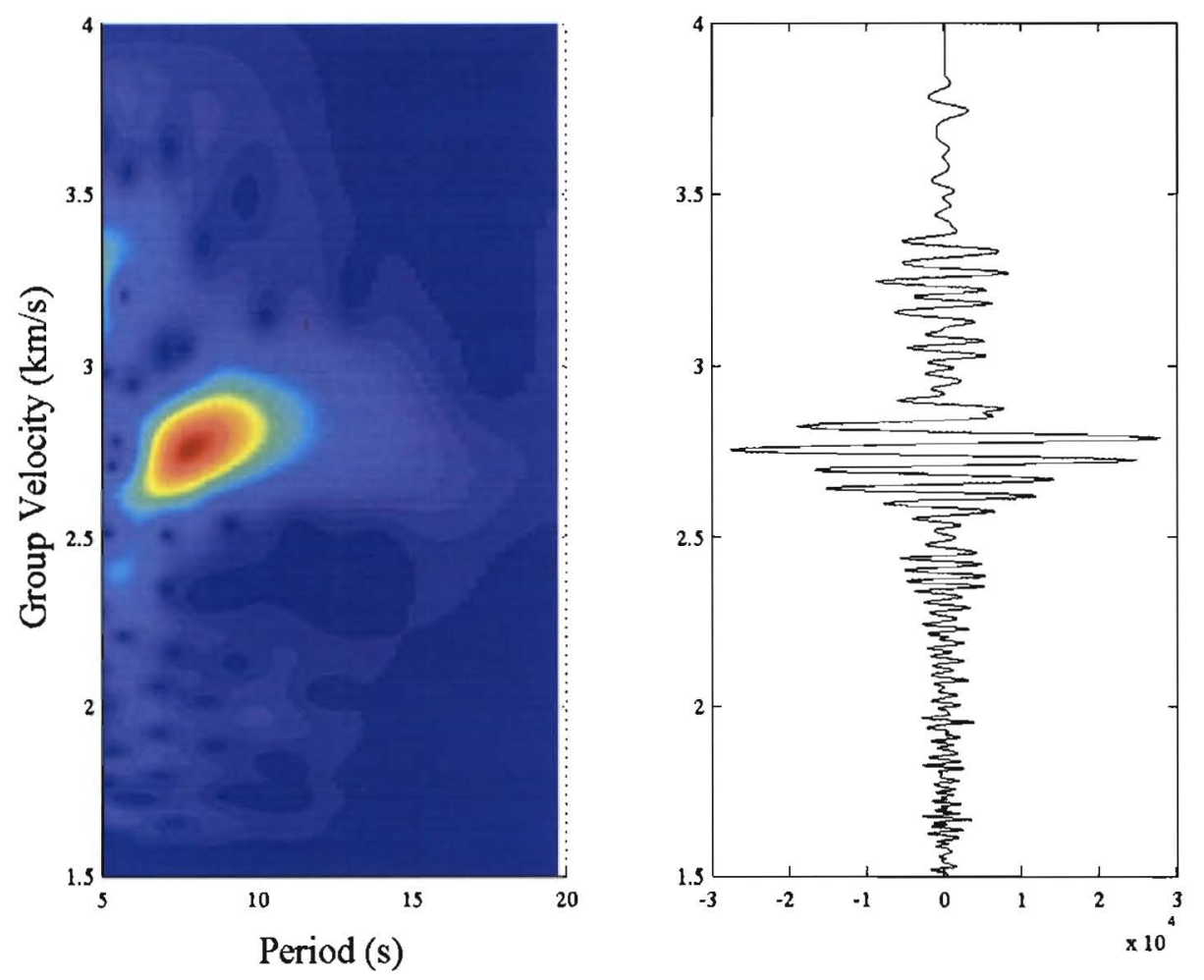

Figure 10: 


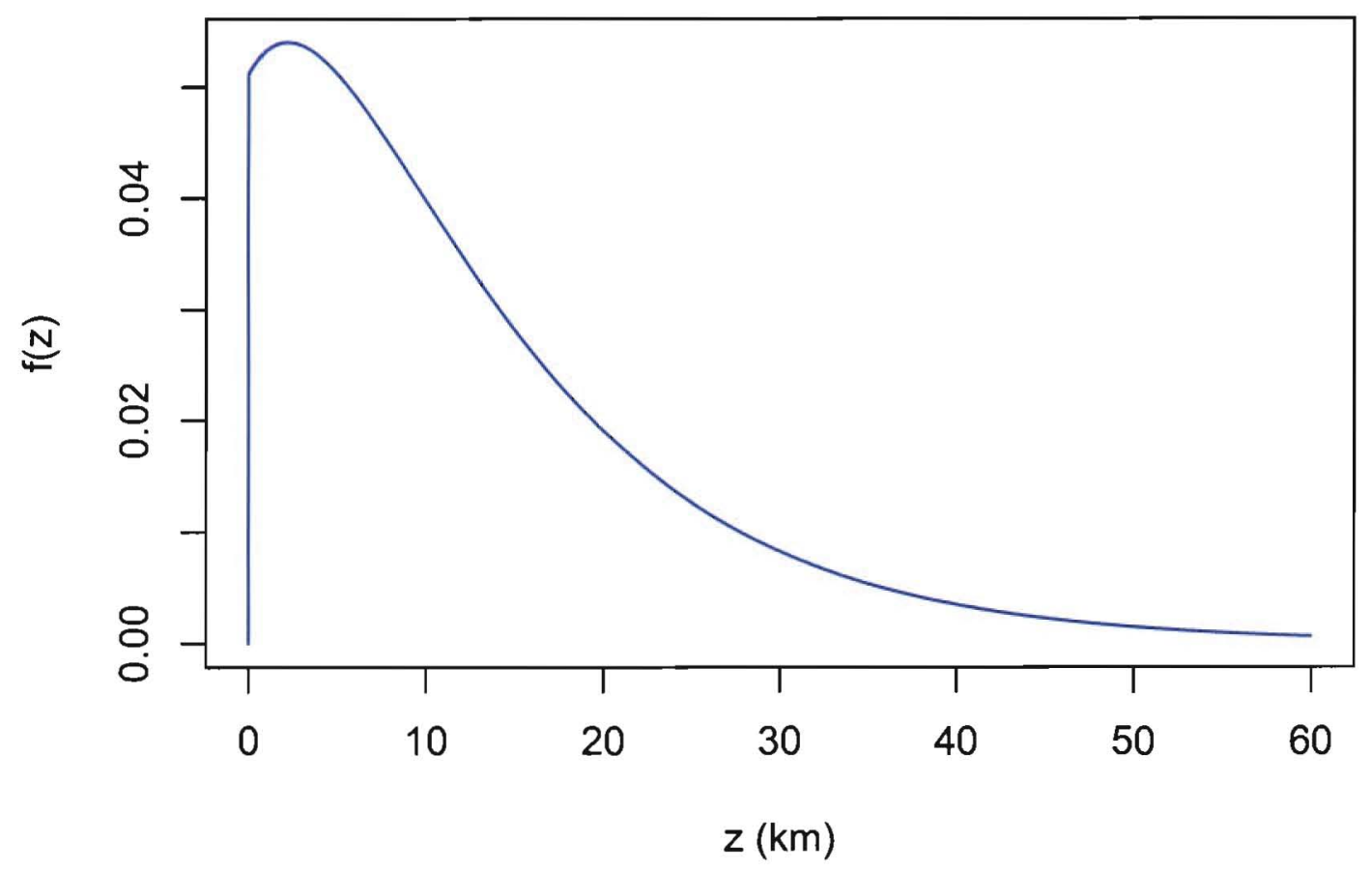

Figure 11: 


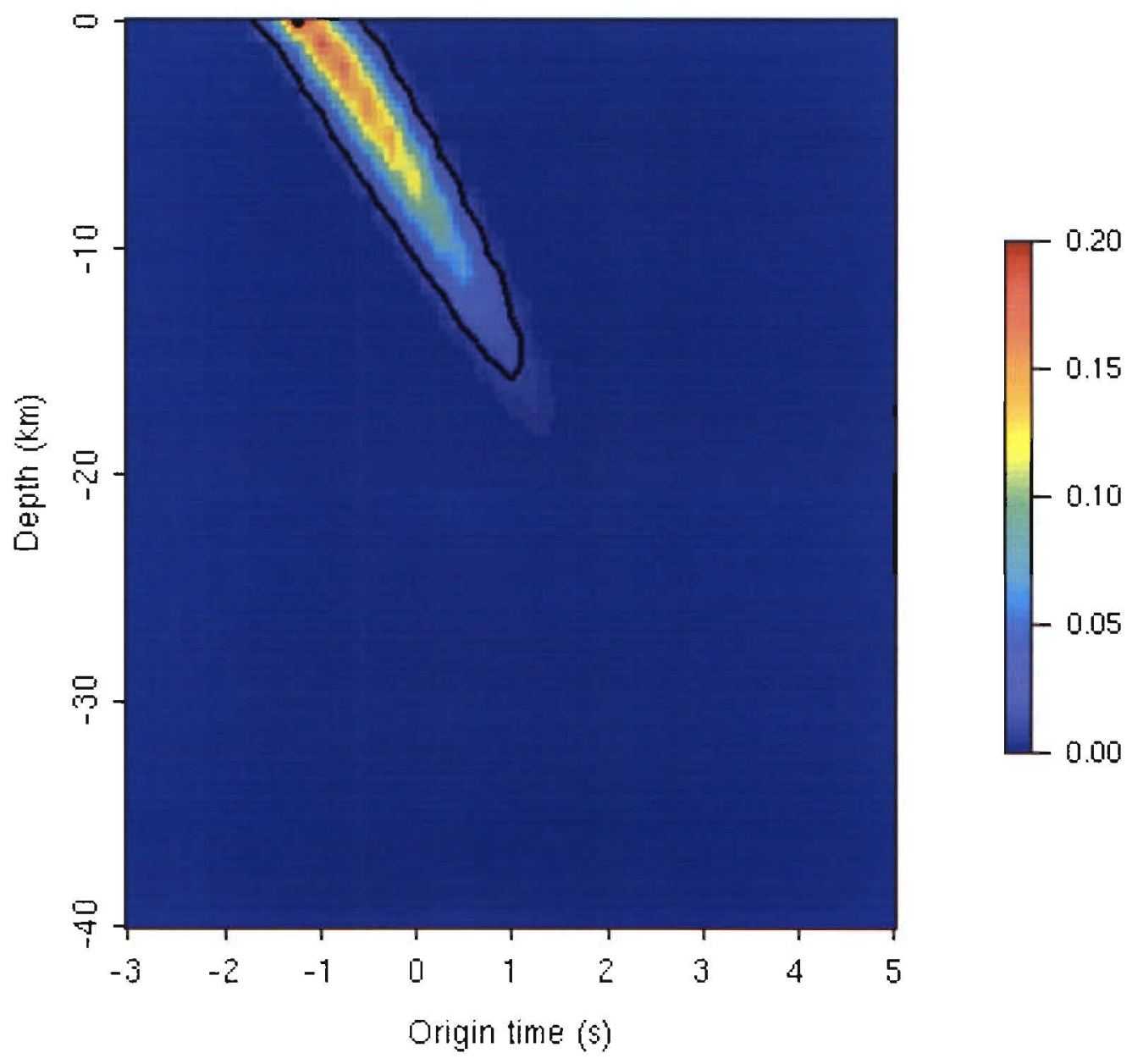

Figure 12: 


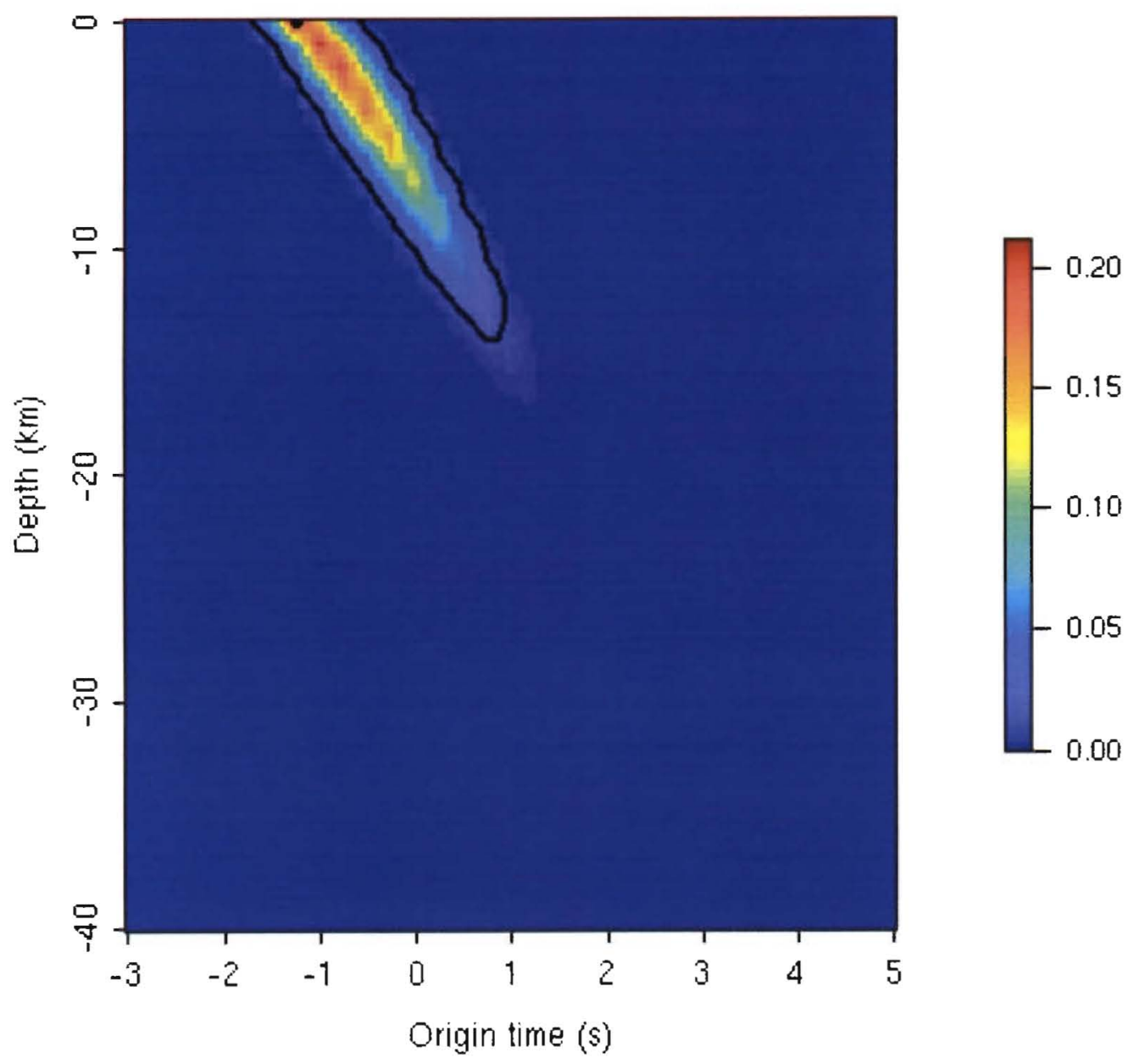

Figure 13: 


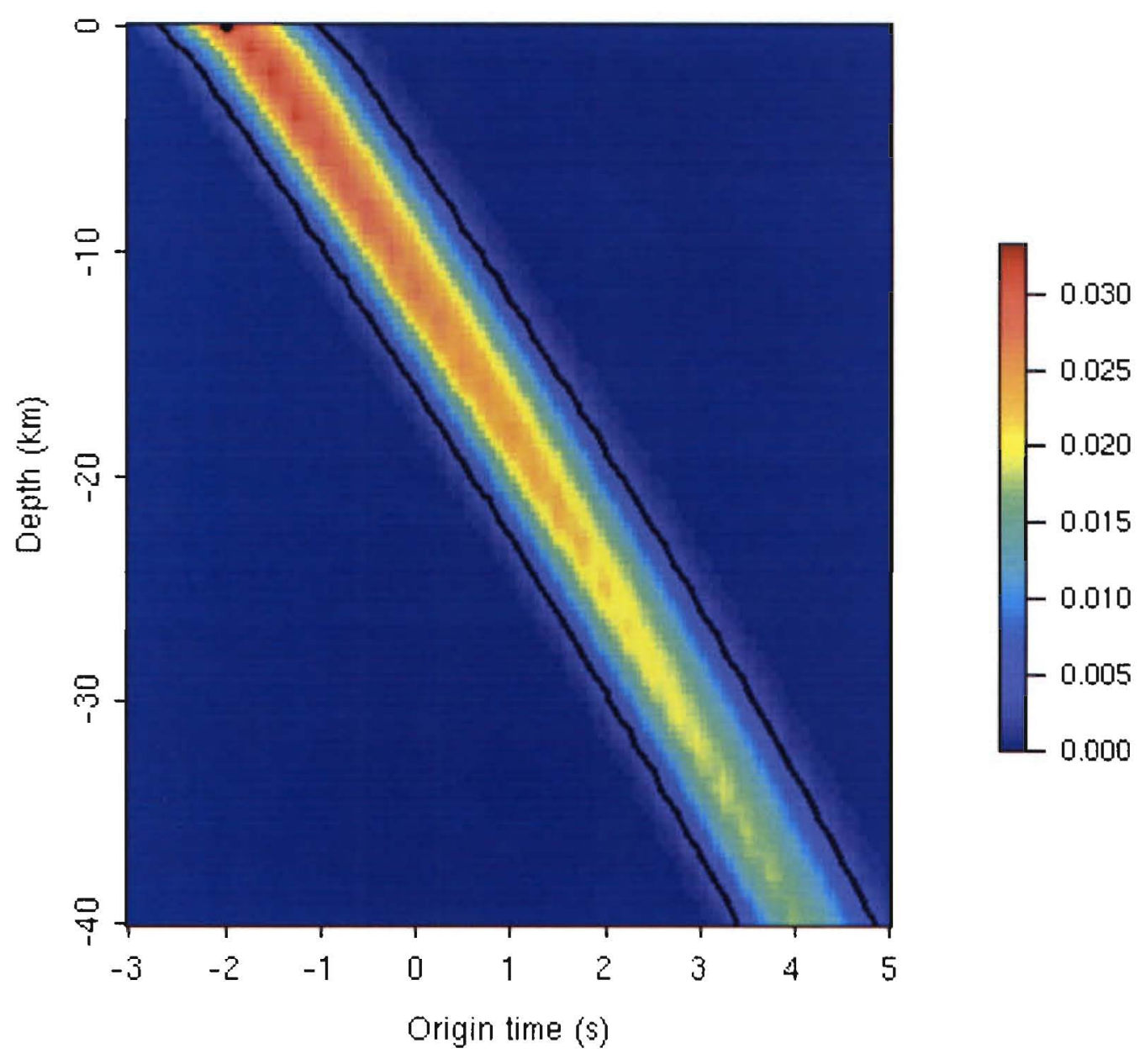

Figure 14: 


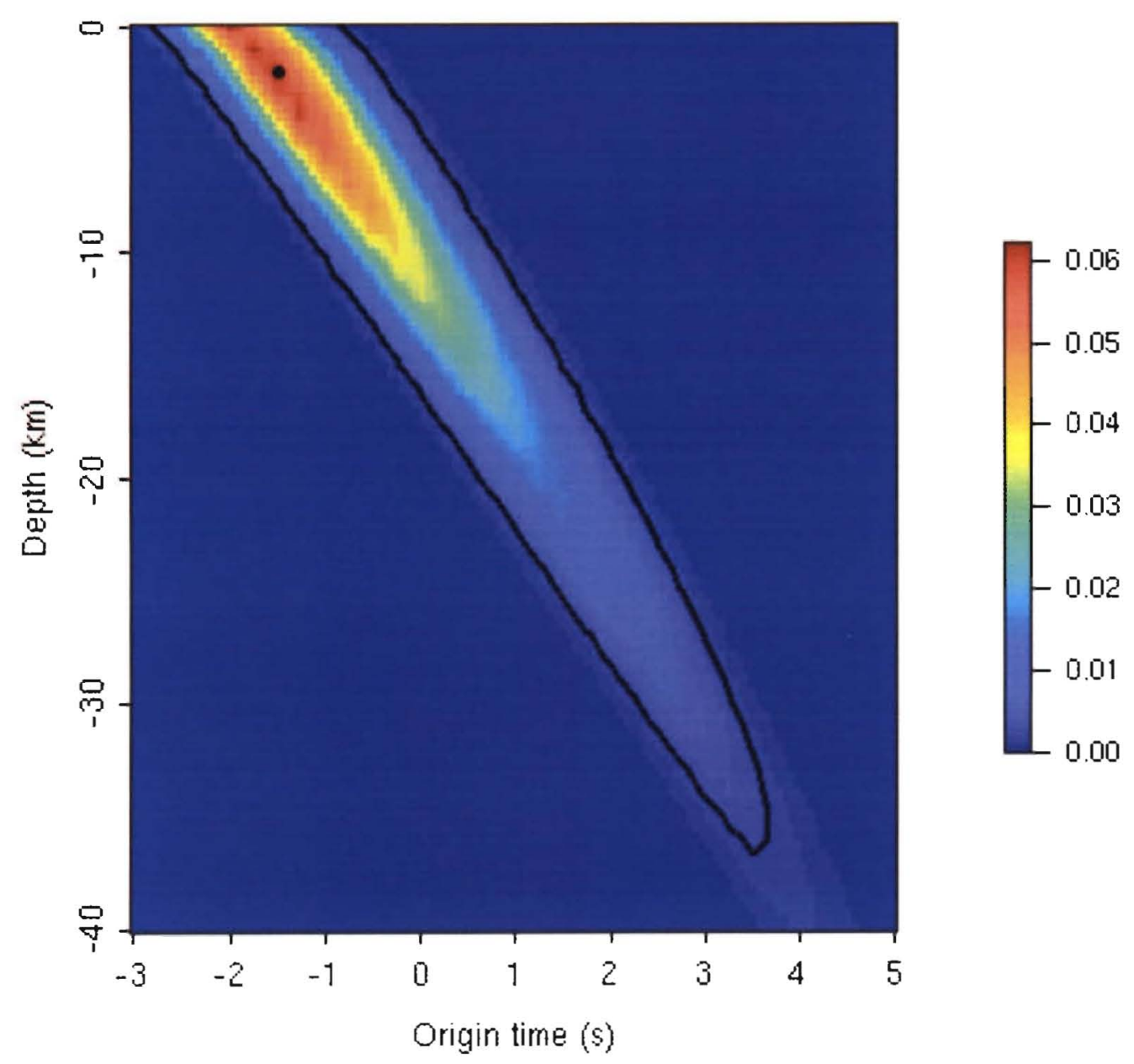

Figure 15: 